\title{
TITLE:
}

\section{CONSTRUCTIONS FOR CYCLIC STEINER 2-DESIGNS}

$\operatorname{AUTHOR}(\mathrm{S})$ :

Mathon, Rudolf

\section{CITATION:}

Mathon, Rudolf. CONSTRUCTIONS FOR CYCLIC STEINER 2-DESIGNS. 数 理解析研究所講究録 1987, 607: 22-32

ISSUE DATE:

1987-02

URL:

http://hdl.handle.net/2433/99708

RIGHT: 


\title{
CONSTRUCTIONS FOR CYCLIC STEINER 2-DESIGNS
}

\author{
Rudolf Mathon* \\ Department of Computer Science \\ University of Toronto \\ Toronto, Ontario, Canada M5S 1A4
}

\begin{abstract}
This paper surveys direct and recursive constructions for cyclic Steiner 2designs. A new method is presented for cyclic designs with blocks having a prime number of elements. Several new constructions are given for designs with block size 4 which are based on perfect systems of difference sets and additive sequences of permutations.
\end{abstract}

\section{Introduction}

A balanced incomplete block design (briefly BIBD) with parameters $(v, k, \lambda)$ is a pair $(V, B)$ where $V$ is a $v$-set and $B$ is a collection of $k$-subsets of $V$ (called blocks) such that every 2subset of $V$ is contained in exactly $\lambda$ blocks. A Steiner 2 -design is a $(v, k, \lambda)$ BIBD with $\lambda=1$. An automorphism of a BIBD $(V, B)$ is a bijection $\phi: V \rightarrow V$ such that the induced mapping $\Phi: B \rightarrow B$ is also a bijection. The set of all such mappings forms a group under composition called the automorphism group of the design.

$\mathrm{A}(v, k, \lambda) \mathrm{BIBD}$ is cyclic if it has an automorphism consisting of a single cycle of length $v$. Cyclic $(v, k, \lambda)$ BIBD's will be denoted by $C(v, k, \lambda)$. A $(v, k, \lambda)$ difference family (briefly DF) is a collection of $k$-subsets $D_{1}, \ldots, D_{t}$ of the integers $Z_{v}$ modulo $v$ such that for each nonzero $x \in Z_{v}$ the congruence $d_{i}-d_{j} \equiv x(\bmod v)$ has exactly $\lambda$ solution pairs $\left(d_{i}, d_{j}\right)$ with $d_{i}, d_{j} \in D_{l}$, for some $l$. A $(v, k, \lambda) \mathrm{DF}$ is called simple if $\lambda=1$. It is easily verified that a necessary condition for the existence of a $(v, k, \lambda) \mathrm{DF}$ is $\lambda(v-1) \equiv 0 \bmod k(k-1)$. In particular, if a simple DF exists then $v \equiv 1 \bmod k(k-1)$. A $(v, k, \lambda)$ DF generates a cyclic BIBD $C(v, k, \lambda)$ with $V=Z_{v}$ and $B=\left\{\sigma^{i} D_{l} \mid 0 \leq i<v, 1 \leq l \leq t\right\}$, where $\sigma: V \rightarrow V, \sigma(x)=x+1 \bmod v$ and $n=\lambda(v-1) l$ $(k(k-1))$. The $t$ blocks $D_{1}, \ldots, D_{t}$ are called starter or base blocks of the design $(V, B)$ (they are representatives of the orbits of $B$ under $\sigma$ ). An orbit analysis of a cyclic Steiner 2-design $C(v, k)$ yields the following necessary existence condition:

$$
v \equiv 1, k \bmod k(k-1) \text {. }
$$

The case $v=k(k-1) t+1$ corresponds to a simple DF. If $v=k(k-1) t+k$ then there are $t+1$ starter blocks $D_{0}, D_{1}, \ldots, D_{t}$, where $D_{0}=\{0, m, 2 m, \ldots,(k-1) m\}, m=(k-1) t+1$

\footnotetext{
* Research supported by NSERC Grant No.A8651.
} 
generates a $m$-orbit and $D_{1}, \ldots, D_{t}$ generate $t v$-orbits under $\sigma$. It is clear, that the differences in $D_{1}, \ldots, D_{t}$ cover the elements $Z_{v} D_{0}$ exactly once.

Two difference families $\mathbf{D}=\left\{D_{1}, \ldots, D_{t}\right\}$ and $\mathbf{D}^{\prime}=\left\{D_{1}^{\prime}, \ldots, D_{t}^{\prime}\right\}$ are said to be equivalent if for some integers $r, s_{1}, \ldots, s_{t}$

$$
\left\{D_{1}^{\prime}, \ldots, D_{t}^{\prime}\right\}=\left\{r D_{1}+s_{1}, \ldots, r D_{t}+s_{t}\right\} \bmod v \text {. }
$$

If $\mathbf{D}$ is equivalent with itself, then the corresponding $r$ is called a multiplier of $\mathbf{D}$ and $\tau: x \rightarrow r x$, $x \in Z_{v}$ is an automorphism of the cyclic design.

Cyclic designs have a nice structure and interesting algebraic properties. Their concise representation makes them attractive in applications and for testing purposes. Cyclic BIBD's and difference systems have been studied by many authors [3], [7], [10], [13]. Results concerning cyclic Steiner 2-designs are surveyed in [5] which also contains a fairly extensive bibliography.

The present paper addresses the problem of existence of cyclic Steiner 2-designs $C(v, k, 1)$. In the next two sections we discuss direct and recursive constructions for general block sizes $k$. In addition to known techniques, several new constructions are presented for $k=4$ and 5 . We conclude with a list of open problems. The paper significantly extends the existence results given in [5] for cyclic Steiner 2-designs with block sizes $k>3$.

\section{Direct Constructions}

The majority of direct methods for constructing cyclic designs are based on finite fields. In this section we survey those constructions which apply to Steiner 2-designs and apply them to generate some new designs with blocks of prime size.

We begin with two general constructions of Wilson for $(v, k, 1)$ difference families [13].

Theorem 1 Let $p=k(k-1) t+1$ be a prime and $\alpha$ a primitive root of $Z_{p}$. Let $H^{m}$ be the multiplicative subgroup of $Z_{p} \backslash\{0\}$ generated by $\alpha^{m}$ and let $\omega=\alpha^{2 m t}$.

(i) If $k=2 m+1$ is odd and $\left\{\omega-1, \omega^{2}-1, \ldots, \omega^{m}-1\right\}$ is a system of representatives for the cosets $\alpha^{i} H^{m}, i=0,1, \ldots, m-1$, then the blocks $D_{i+1}=\left\{\alpha^{m i}, \omega \alpha^{m i}, \ldots, \omega^{2 m} \alpha^{m i}\right\}$, $i=0,1, \ldots, t-1$ form a $(p, k, 1) \mathrm{DF}$.

(ii) If $k=2 m$ is even and $\left\{1, \omega-1, \ldots, \omega^{m-1}-1\right\}$ is a system of representatives for the cosets $\alpha^{i} H^{m}, i=0,1, \ldots, m-1$, then the blocks $D_{i+1}=\left\{0, \alpha^{m i}, \omega \alpha^{m i}, \ldots, \omega^{2 m-2} \alpha^{m i}\right\}$, $i=0,1, \ldots, t-1$ form a $(p, k, 1) \mathrm{DF}$ in $Z_{p}$.

Theorem 2 Let $p=k(k-1) t+1$ be a prime and $\alpha$ a primitive root of $Z_{p}$. If there exists a set $B=\left\{b_{1}, \ldots, b_{k}\right\} \subset Z_{p}$ such that $\left\{b_{j}-b_{i} \mid 1 \leq i<j \leq k\right\}$ is a system of representatives for the cosets $\alpha^{i} H^{m}, i=0,1, \ldots, m-1$, where $m=k(k-1) / 2$ and $H^{m}$ is the subgroup of $Z_{p} \backslash\{0\}$ generated by $\alpha^{m}$, then $D_{i+1}=\alpha^{2 m i} B, i=0,1, \ldots, t-1$ is a $(p, k, 1)$ DF in $Z_{p}$. 
Our next result concerns the case $v \equiv k \bmod k(k-1)$.

Theorem 3 Let $k=2 m+1$ and $p=2 m t+1, n \geq 2$ be two odd primes and let $\alpha$ be a primitive root of $Z_{p}$. Define $m-1$ numbers $r_{i}$ by the equations $\alpha^{r_{i}}=\alpha^{t i}-1, i=1, \ldots, m-1$. If there exists a $\beta \in Z_{k}$ such that the $2 m$ elements $\pm 1, \pm\left(\beta^{i i}-1\right) \beta^{-r_{i}}, i=1, \ldots, m-1$ are all distinct in $Z_{k}$, then the blocks

$$
\begin{aligned}
& D_{0}=\left\{0_{0}, 0_{1}, \ldots, 0_{2 m}\right\} \\
& D_{i+1}=\left\{0_{0}, \alpha_{\beta^{\prime}}, \alpha_{\beta} t_{i}, \ldots, \alpha^{2 m_{i}-t+t_{i+i}}\right\}, \quad i=0,1, \ldots, t-1
\end{aligned}
$$

form a $(k p, k, 1) \mathrm{DF}$ in $Z_{k p}$.

Proof We note, that since in the family of blocks $\mathbf{B}=\left\{B_{1}, \ldots, B_{t}\right\}, B_{i+1}=\left\{0, \alpha^{i}, \ldots\right.$, $\left.\alpha^{2 m t-t+i}\right\}$ each nonzero difference appears exactly $k-2 m+1$ times, B forms a $(p, k, k)$ DF in $Z_{p}$. To complete the proof, it suffices to show that for any fixed difference in $\mathbf{B}$ the corresponding subscript differences cover every non-zero element of $Z_{k}$ exactly once. Since for each $i$, $\left(\alpha^{t i}-1\right) \alpha^{-r_{i}}=1$ this is equivalent to the assumption that $\pm 1, \pm\left(\beta^{t i}-1\right) \beta^{-r_{i}}, i=1, \ldots, m-1$ are distinct in $Z_{k}$. Finally, since $k$ and $p$ are distinct primes the design is cyclic in $Z_{k p}$.

We will apply Theorem 3 to blocksize $k=7$. Then $m=3$ and $p$ is a prime of the form $p=6 t+1, t \geq 2$. If $\alpha$ is a primitive root of $Z_{p}$, then $\alpha^{3 t}=-1$ and since

$$
\left(\alpha^{t}+1\right) \alpha^{2 t}=\alpha^{2 t}-1=\left(\alpha^{t}+1\right)\left(\alpha^{t}-1\right)
$$

we have $\alpha^{t}-1=\alpha^{2 t}$. Let $r$ be the solution of $\alpha^{r}=\alpha^{2 t}-1$. We require that for some $\beta \in Z_{7}$ the 6 numbers

$$
\pm \beta^{2 t}, \pm\left(\beta^{t}-1\right), \pm \beta^{2 t-r}\left(\beta^{2 t}-1\right)
$$

cover the non-zero elements of $Z_{7}$. Since $\beta^{2 t}$ cannot be congruent to 1 modulo 7 , we see that $t \equiv 1$ or $2 \bmod 3$. If $t \equiv 1 \bmod 3$, then (4) are distinct if either $\beta=2$ and $r \equiv 0 \bmod 3$, or $\beta=4$ and $r \equiv 2 \bmod 3$. If $t \equiv 2 \bmod 3$, then we need either $\beta=2$ and $r \equiv 1 \bmod 3$, or $\beta=4$ and $r \equiv 0$ mod 3. Combining all these conditions we obtain the following result.

Corollary 4 Let $p=6 t+1$ be a prime, $t \geq 2, t \neq 0 \bmod 3$, and let $\alpha$ be a primitive root in $Z_{p}$. Then the blocks (3) form a $(7 p, 7,1)$ DF for some $\beta \in Z_{7}$ if and only if $t \pm r \bmod 3$, where $r$ satisfies $\alpha^{r}=\alpha^{2 t}-1$.

We note, that for some values of $t$ we obtain two non-isomorphic cyclic designs. If $t \equiv 4$ $\bmod 6$, then (4) are distinct also if either $\beta=3$ and $r \equiv 2 \bmod 3$, or $\beta=5$ and $r \equiv 0 \bmod 3$. If $t \equiv 2 \bmod 6$, then (4) are distinct also if either $\beta=3$ and $r \equiv 0 \bmod 3$, or $\beta=5$ and $r \equiv 1 \bmod 3$. 
For $k=7$ solutions exist when $t=2 *, 5,7,13,16^{*}, 26^{*}, 35,37,38^{*}, 40^{*}, 46^{*}$, 47, etc. The base blocks for $t=2 *, 5$ and 7 are

\begin{tabular}{|c|c|c|c|c|c|c|c|c|c|c|c|c|c|}
\hline $0_{0}$ & $1_{1}$ & $4_{4}$ & $3_{2}$ & $12_{1}$ & $9_{4}$ & $10_{2}$ & $0_{0}$ & $1_{1}$ & $4_{4}$ & $3_{2}$ & $12_{1}$ & $9_{4}$ & $10_{2}$ \\
\hline $0_{0}$ & 22 & $8_{1}$ & $6_{4}$ & $11_{2}$ & $5_{1}$ & $7_{4}$ & $0_{0}$ & 25 & $8_{6}$ & $\sigma_{3}$ & $11_{5}$ & $5_{6}$ & $7_{3}$ \\
\hline $0_{0}$ & $1_{1}$ & $26_{4}$ & $25_{2}$ & $30_{1}$ & $5_{4}$ & $\sigma_{2}$ & $0_{0}$ & $1_{1}$ & $37_{2}$ & $36_{4}$ & $42_{1}$ & $\sigma_{2}$ & $7_{4}$ \\
\hline $0_{0}$ & $3_{2}$ & $16_{1}$ & $13_{4}$ & $28_{2}$ & $15_{1}$ & $18_{4}$ & $0_{0}$ & $3_{2}$ & $25_{4}$ & $22_{1}$ & $40_{2}$ & $18_{4}$ & $21_{1}$ \\
\hline $0_{0}$ & $9_{4}$ & $17_{2}$ & $8_{1}$ & $22_{4}$ & $14_{2}$ & $23_{1}$ & $0_{0}$ & $9_{4}$ & $32_{1}$ & $23_{2}$ & $34_{4}$ & $11_{1}$ & $2 \mathrm{O}_{2}$ \\
\hline $0_{0}$ & $27_{1}$ & $20_{4}$ & $24_{2}$ & $4_{1}$ & $11_{4}$ & $7_{2}$ & $0_{0}$ & $27_{1}$ & $10_{2}$ & $26_{4}$ & $16_{1}$ & $33_{2}$ & $17_{4}$ \\
\hline \multirow[t]{3}{*}{$0_{0}$} & $19_{2}$ & $29_{1}$ & $10_{4}$ & $12_{2}$ & $2_{1}$ & $21_{4}$ & $0_{0}$ & $38_{2}$ & $30_{4}$ & $35_{1}$ & $5_{2}$ & $13_{4}$ & $8_{1}$ \\
\hline & & & & & & & $0_{0}$ & $28_{4}$ & $4_{1}$ & 192 & $15_{4}$ & $39_{1}$ & $24_{2}$ \\
\hline & & & & & & & $0_{0}$ & $41_{1}$ & $12_{2}$ & $14_{4}$ & $2{ }_{1}$ & $31_{2}$ & $29_{4}$ \\
\hline
\end{tabular}

The solutions for $t=5$ and 7 are first examples of BIBD's with the parameters $(217,7,1)$ and $(301,7,1)$, respectively. For $k=11$ solutions exist when $t=33,54^{*}, 57,91,94^{*}$, etc. and for $k=13, t=13,19,59$, etc. (* indicates 2 solutions).

We conclude this section with a well-known result in finite geometries [6].

Theorem 5 Let $q$ be a prime power. Then the lines in the projective geometry $P G(n, q), n \geq 2$ form a cyclic design with parameters $\left(\left(q^{n+1}-1\right) /(q-1), q+1,1\right)$.

\section{Recursive Constructions}

Given two difference families it is sometimes possible to combine them to construct a new one. Several such constructions are known for general cyclic BIBD's [4] [8] [14]. To apply them, various conditions on the block sizes are usually required.

We begin with a construction by C.J. Colbourn and M.J. Colbourn [4].

Theorem 6 Let $A^{t}{ }_{i}=\left\{0, a^{i}{ }_{1}, \ldots, a^{i}{ }_{k-1}\right\}, i=1, \ldots, t$ be a $(v, k, 1) \mathrm{DF}$ in $Z_{v}$ and let $B^{s_{j}}=$ $\left\{0, b_{1}^{j_{1}}, \ldots, b^{j_{k-1}}\right\}, j=1, \ldots, s$ be a $(w, k, 1) \mathrm{DF}$ in $Z_{w}$.

(i) If $v=k(k-1) t+1$ and $w$ is relatively prime to $(k-1)$ !, then for $i=1, \ldots, t$, $j=1, \ldots, s$ and $l=0,1, \ldots, w-1$

$$
\left.\begin{array}{l}
\left\{0, a^{i}+l v, a_{2}{ }_{2}+2 l v, \ldots, a_{k-1}^{i_{k}}+(k-1) l v\right\} \\
\left\{0, v b^{j_{1}}, v b j_{i}, \ldots, v b j_{k-1}\right\}
\end{array}\right\}
$$

is a $(v w, k, 1)$ DF in $Z_{v w}$. 
(ii) If $v=k \alpha, w=k \beta$ and $\beta$ is relatively prime to $(k-1)$ !, then for $i=1, \ldots, t, j=1, \ldots, s$ and $l=0,1, \ldots, w-1$

$$
\left.\begin{array}{l}
\left\{0, a_{1} i_{1}+l v, a_{2} i_{2}+2 l v, \ldots, a_{k-1}+(k-1) l v\right\} \\
\left\{0, \alpha b j_{1}, \alpha b j_{2}, \ldots, \alpha b k_{k-1}\right\} \\
\{0, \alpha \beta, 2 \alpha \beta, \ldots,(k-1) \alpha \beta\}
\end{array}\right\}
$$

is a $(k \alpha \beta, k, 1) \mathrm{DF}$ in $Z_{k \alpha \beta}$. Here $\alpha=(k-1) t+1, \beta=(k-1) s+1$, and only full orbit base blocks $A^{t}{ }_{i}, B_{j}$ are considered.

We note that the construction can be used if either $w$ or $\beta$ are prime. Then the existence of a $(w, k, 1)$ DF implies the existence of a $\left(w^{n}, k, 1\right)$ DF for every $n \geq 1$. Similarly, from a $(k \beta, k, 1)$ $\mathrm{DF}$ we obtain a $\left(k \beta^{n}, k, 1\right) \mathrm{DF}$. Also, if a $(v, k, 1) \mathrm{DF}$ exists with $v \equiv 1 \bmod k(k-1)$ and prime $k$ then there exists a $(v k, k, 1) \mathrm{DF}$.

In [8] M. Jimbo and S. Kuriki have introduced a more general construction for cyclic BIBD's which is based on orthogonal arrays. Applying it to Steiner 2-designs we obtain the following typical result.

Theorem 7 Suppose there exists a $C(v, k, 1)$ and a $C(w, k, 1)$, where $v \equiv 1 \bmod k(k-1)$ and $k$ is an odd prime. Then there exists a $C(v w, k, 1)$. If, in addition, $w \equiv 1 \bmod k(k-1)$, then the conclusion holds for $k$ a prime power.

So, for example, if $k$ is an odd prime not dividing $v$, then the existence of a $C(v, k, 1)$ implies the existence of both $C\left(v^{n}, k, 1\right)$ and $C\left(k v^{n}, k, 1\right)$ for any $n \geq 1$.

The next construction employs cyclic pairwise balanced designs. A pairwise balanced design (briefly $\mathrm{PBD}$ ) is a pair $(V, B)$ where $V$ is a $v$-set and $B$ is a collection of subsets of $V$ (blocks) such that every 2-subset of $V$ is contained in exactly one block. A PBD will be denoted by $(v, K, 1)$, where $K=\left\{k_{1}, \ldots, k_{n}\right\}$ is the set of block sizes.

Theorem 8 Suppose there exists a cyclic $(v, K, 1)$ PBD with $K=\left\{k_{1}, \ldots, k_{n}\right\}$ and that for each $k_{i}$ there exists a $\left(k_{i}, k, 1\right)$ Steiner 2-design. Then there exists a $C(v, k, 1)$.

Proof Replace each base block in the PBD by the blocks of the corresponding Steiner 2-design to obtain the base blocks of the final $C(v, k, 1)$.

In the next section we shall give some other recursive constructions for cyclic designs with blocks of size 4 and 5 which are based on the concepts of perfect systems of difference sets and additive sequences of permutations. 


\section{Special Constructions}

The existence question for cyclic Steiner triple systems has been completely settled by Peltesohn [10], who constructed $C(v, 3,1)$ for all $v \equiv 1,3 \bmod 6, v \neq 9$.

For block sizes $k>3$ the existence problem for $C(v, k, 1)$ remains unsolved. The state of affairs is most promising for the cases $k=4$ and 5 .

In order to present additional recursive constructions we require a few more definitions.

A collection of $t k$-subsets $D_{i}=\left\{d^{i_{0}}, d^{i_{1}}, \ldots, d^{i}{ }_{k-1}\right\}, 0=d^{i}{ }_{0}<d^{i_{1}}<\cdots<d^{i}{ }_{k-1}$, $i=1, \ldots, t$ is said to be a perfect difference family (PDF) in $Z_{v}, v=k(k-) t+1$, if the $t k(k-1) / 2$ differences $d^{i}{ }_{l}-d^{i}{ }_{j}, 0 \leq j<l<k$ cover the set $\{1,2, \ldots, t k(k-1) / 2\}$. PDF's are equivalent to regular perfect systems of difference sets starting with 1 , which have been studied by many authors (see [1] for a recent survey). It has been shown [2] that PDF's can exist only when $k$ is 3,4 or 5 . For $k=3$ the existence of a PDF is related to Skolem's partitioning problem [1].

Let $X^{1}$ be the $m$-vector $(-r,-r+1, \ldots,-1,0,1, \ldots, r-1, r), m=2 r+1$ and let $X^{2}, \ldots, X^{n}$ be permutations of $X^{1}$. Then $X^{1}, \ldots, X^{n}$ is an additive sequence of permutations (ASP) of order $m$ and length $n$ if the vector sum of every subsequence of consecutive permutations is again a permutation of $X^{1}$. ASP's play an important role in recursive constructions for PDF's and vice versa [1] [11] [12].

\section{Block size 4}

We begin with two direct constructions.

Theorem 9 let $p=12 t+1, t \geq 1$ be a prime and let $\alpha$ be a primitive root of $Z_{p}$.

(i) ([3] [13]) If $p \neq x^{2}+36 y^{2}$ for any integers $x$ and $y$ then

$$
\left\{0, \alpha^{2 i}, \alpha^{4 t+2 i}, \alpha^{8 t+2 i}\right\} i=0,1, \ldots, t-1
$$

is a $(p, 4,1) \mathrm{DF}$ in $Z_{p}$.

(ii) ([5]) If $\alpha \equiv 3 \bmod 4$ (and such an $\alpha$ always exists in $Z_{p}$ ) then

$$
\begin{aligned}
& \left.\left\{0, \alpha^{4 i}, \alpha^{4 i+3}, \alpha^{4 i+6}\right\} i=0, \ldots, 3 t-1\right\} \\
& \left.\left\{0, \alpha^{4 j+1}, \alpha^{4 t+4 j+1}, \alpha^{8 t+4 j+1}\right\} j=0, \ldots, t-1\right\} \\
& \{0, p, 2 p, 3 p\}
\end{aligned}
$$

form a $(4 p, 4,1) \mathrm{DF}$ in $Z_{4 p}$.

The next two constructions will exhibit the relationship between PDF's and ASP's.

Theorem 10 ([3] [13]) Let $D_{i}=\left\{0, a_{i}, b_{i}, c_{i}\right\}, i=1, \ldots, t$ be a PDF in $Z_{12 t+1}$ and let $X^{1}, X^{2}, X^{3}$ be an ASP of order $m=2 r+1, r \geq 2$ and length 3 . Then 
(i) For $i=1, \ldots, t$ and $j=1, \ldots, m$ the $6 t m$ positive differences in the family

$$
\Delta_{m i-m+j}=\left\{0, m a_{i}+\alpha_{j}, m b_{i}+\beta_{j}, m c_{i}+\gamma_{j}\right\}
$$

cover the set $\{r+1, r+2, \ldots, r+6 t m\}$. Here $\alpha, \beta$ and $\gamma$ are the $m$-vectors $X^{1}, X^{1}+X^{2}$, $X^{1}+X^{2}+X^{3}$, respectively.

(ii) For $i=1, \ldots, t$

$$
\left.\begin{array}{rl}
X_{i}{ }^{1} & =(-c, a-c,-b, b-c, a-b,-a, a, b-a, c-b, b, c-a, c)_{i} \\
X_{i}^{2} & =(c-b, c, b-a, c-a, b-c, a-c,-b, a, b,-c, a-b,-a)_{i} \\
X_{i}{ }^{3} & =(b-a,-b, a-c, a, c, c-b, b-c,-c,-a, c-a, b, a-b)_{i}
\end{array}\right\}
$$

the $(12 t+1)$-vectors $X^{j}=\left(0, X_{1}^{j}, \ldots, X_{t}^{j}\right), j=1,2,3$ form an ASP of order $12 t+1$ and length 3 .

In order to utilize products of the form (9) for constructing new difference families we need to find additional base blocks with differences covering the set $\{1, \ldots, r\}$ and possibly $\{r+6 t m+1, \ldots, 6 x\}$ for some $x \geq 1$.

We list now the known recursive constructions for $1 \leq m \leq 25$.

Theorem 11 Let $D(t)=\left\{D_{1}, \ldots, D_{t}\right\}$ be a PDF and let $\Delta(m t)=\left\{\Delta_{1}, \ldots, \Delta_{m t}\right\}$ be defined by (9), where $m=2 r+1$ and $\alpha=(-r,-r+1, \cdots,-1,0,1, \ldots, r-1, r)$. Then

1. For $r=2$

$$
\begin{aligned}
\beta=(-2,0,2,-1,1), \quad \gamma=(0,-2,1,-1,2) \\
D(5 t+1)=\Delta(5 t) \cup\{0,1,30 t+4,30 t+6\}
\end{aligned}
$$

is a PDF in $Z_{60 t+13}$.

2. For $r=3$

$$
\begin{gathered}
\beta=(-1,-2,-3,3,2,1,0), \quad \gamma=(-2,1,-3,0,3,-1,2) \\
D(7 t+1)=\Delta(7 t) \cup\{0,2,3,42 t+7\}
\end{gathered}
$$

is a $D F$ in $Z_{84 t+13}$.

3. For $r=6$

$$
\begin{gathered}
\beta=(-4,-5,-1,-2,3,-6,6,5,1,-3,0,2,4) \\
\gamma=(-1,-5,-6,3,-3,-4,4,2,5,-2,6,1,0) \\
\quad D(13 t+1)=\Delta(13 t) \cup\{0,1,4,6\}
\end{gathered}
$$

is a PDF in $Z_{156 t+13}$.

4. For $r=9$

$$
\beta=(-6,-7,1,-2,-3,5,3,-9,-4,7,-8,0,-5,9,-1,6,2,4,8)
$$




$$
\begin{aligned}
& \gamma=(-2,1,-8,-9,3,-1,-5,-6,5,2,-7,7,-3,8,-4,6,0,9,4) \\
& D(19 t+4)= \Delta(19 t) \cup\{0,1,7, x+23\} \cup\{0,2, x+14, x+19\} \\
& \cup\{0,3, x+13, x+21\} \cup\{0,4, x+15, x+24\}, x=114 t
\end{aligned}
$$

is a PDF in $Z_{228 t+49}$.

5. For $r=11$

$$
\begin{gathered}
\beta=(0,-2,1,-7,2,-6,-1,-5,4,-10,-11,-9,6,-4,-8,-3,11,8,10,3,5,7,9) \\
\gamma=(9,5,4,-10,0,-7,10,-9,8,-4,-3,1,-5,-11,-8,2,6,-2,11,-6,7,-1,3) \\
D(23 t+5)=\Delta(23 t) \cup\{0,1,8, x+28\} \cup\{0,2, x+14, x+24\} \cup \\
\{0,3, x+18, x+29\} \cup\{0,4, x+17, x+23\} \cup\{0,5, x+21, x+30\}, x=138 t
\end{gathered}
$$

is a PDF in $Z_{276 t+61}$.

6. For $r=12$

using $\alpha, \beta, \gamma$ and $\Delta(5 t)$ from 1 to obtain $\Delta(25 t)$

$$
\begin{aligned}
D(25 t+5) & =\Delta(25 t) \cup\{0,1, x+18, x+29\} \cup\{0,4, x+20, x+26\} \\
& \cup\{0,3,8, x+27\} \cup\{0,7, x+21, x+30\} \cup\{0,10,12, x+25\}, x=150 t
\end{aligned}
$$

is a PDF in $Z_{300 t+61}$, and

$$
\begin{aligned}
D(25 t+6) & =\Delta(25 t) \cup\{0,1, x+34, x+36\} \cup\{0,3, x+18, x+29\} \\
& \cup\{0,4, x+20, x+28\} \cup\{0,5, x+22, x+32\} \\
& \cup\{0,6, x+19, x+31\} \cup\{0,7, x+21, x+30\}, x=150 t
\end{aligned}
$$

is a PDF in $Z_{300 t+79}$.

Proof Use (9) to check that the required sets are covered by all differences from the base blocks.

We note that the constructions $2,5,6 \mathrm{~b}$ are new and that $1,3,4,6 \mathrm{a}$ have been known [11]. The ASP with $r=11$ has been found by P.J. Laufer.

If we apply all methods listed in Sections 2,3 and 4 and add the computer generated DF's from [5] we obtain the following results for $1 \leq t \leq 50$ :

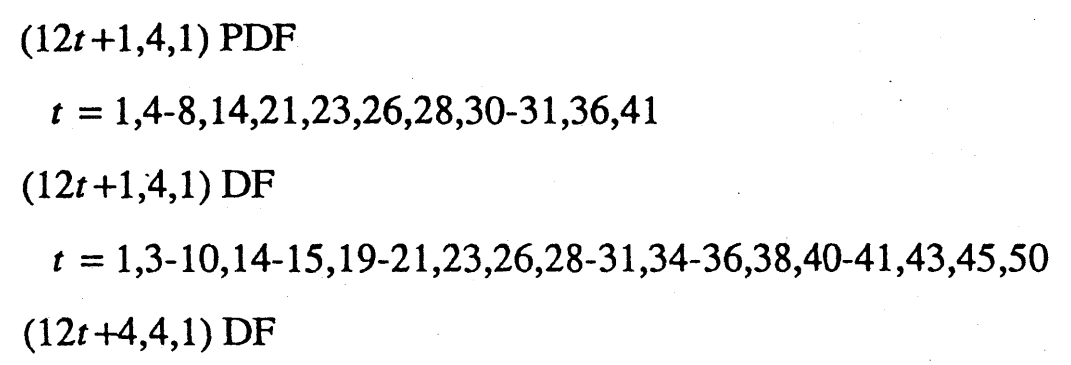


$t=3-6,12,20,24,30,32,36,43$.

\section{Block size 5}

As before, two direct constructions are known.

Theorem 12 Let $p=20 t+1, t \geq 1$ be a prime and let $\alpha$ be a primitive root of $Z_{p}$.

(i) ([3] [13]) If $p \neq x^{2}+100 y^{2}$ for any integers $x$ and $y$ then

$$
\left\{\alpha^{2 i}, \alpha^{4 t+2 i}, \alpha^{8 t+2 i}, \alpha^{12 t+2 i}, \alpha^{16 t+2 i}\right\} \quad i=0,1, \ldots, t-1
$$

is a $(p, 5,1) \mathrm{DF}$ in $Z_{p}$.

(ii) ([5]) If $\alpha^{r}+1=\alpha^{s}\left(\alpha^{r}-1\right)$ for some odd integers $r$ and $s$ then

$$
\begin{aligned}
& \left\{0, \alpha^{2 i}, \alpha^{2 i+r}, \alpha^{2 t+2 i}, \alpha^{2 t+2 i+r}\right\} \quad i=0,1, \ldots, t-1 \\
& \{0, p, 2 p, 3 p, 4 p\}
\end{aligned}
$$

form a $(5 p, 5,1) \mathrm{DF}$ in $Z_{5 p}$.

Concerning PDF's with blocks of size 5 and ASP of length 4, results can be proved which are similar to those stated in Theorem 10 [1]. They can be used to derive the following construction.

Theorem 13 Let $D(t)=\left\{D_{1}, \ldots, D_{t}\right\}$ be a PDF in $C_{20 t+1}$ and let $D(s)=\left\{D_{1}, \ldots, D_{s}\right\}$ be a DF in $C_{20 s+1}$. Then a DF $D(r)$ exists in $C_{20 r+1}, r=20 s t+s+t$ and $D(r)$ is perfect whenever $D(s)$ is perfect.

Proof Use $D(t)$ to construct an ASP of length 4 and order $m=20 t+1$ [1]. With help of this ASP construct the blocks $\Delta(m s)$ in a similar way as in (9). Then $D(r)=\Delta(m s) \cup D(s)$.

PDF's with $k=5$ can exist only if $t$ is even and $t \geq 6$ [1]. They have been enumerated for $t=6$ [9] and examples are known for $t=8,10,732,974$, etc.

Difference families are known for the following values of $t, 1 \leq t \leq 50$ :

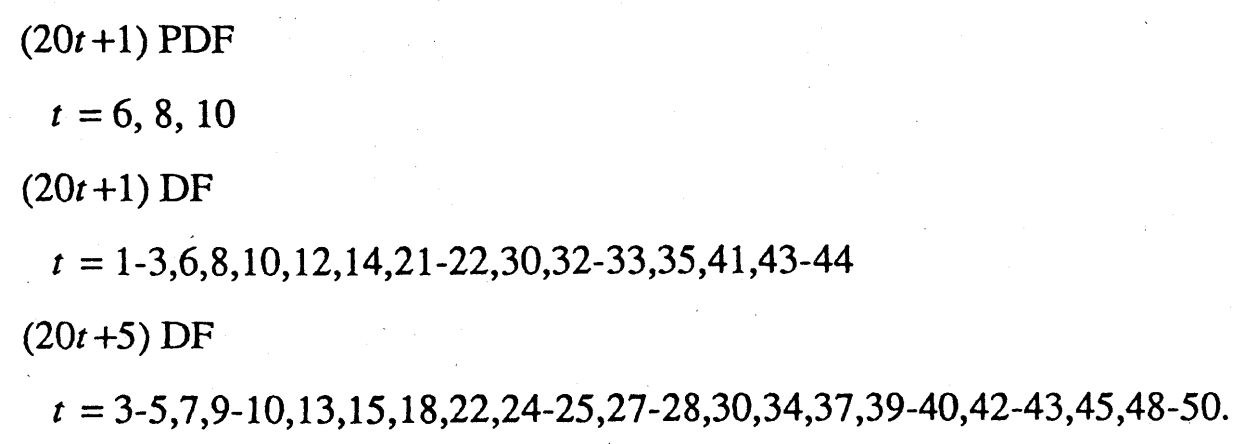




\section{Open Problems}

1. Does there exist a $(12 t+1,4,1)$ DF for every $t \geq 3$ ? Can all of these DF's be perfect if $t \geq 4$ ?

2. Does there exist a $C(v, 4,1)$ for every $v \neq 16,25$ and 28 ?

3. Does there exist an ASP of length 3 for every order $m \geq 5, m \neq 9,10$ ?

4. Do there exist $C(v, 5,1)$ for $v=81$ and 85 ?

5. Construct examples of PDF's $D(t)$ for $k=5$ and even $t \geq 12$.

6. Construct examples of ASP of length 4 for orders $m \geq 7$. 


\section{References}

[1] J. Abrham, Perfect systems of difference sets - a survey, Ars Combinatoria, 17A (1984), 5-36.

[2] J.-C. Bermond, A. Kotzig, J. Turgeon, On a combinatorial problem of antennas in radioastronomy, Proc. 18th Hungarian Combinatorial Colloquium, North Holland, 1976, 135-149.

[3] R.C. Bose, On the construction of balanced incomplete block designs, Ann. Eugenics 9 (1939), 353-399.

[4] M.J. Colbourn, C.J. Colbourn, On cyclic block designs, Math. Report of Canadian Academy of Science 2 (1980), 21-26.

[5] M.J. Colbourn, R.A. Mathon, On cyclic Steiner 2-designs, Ann. Discrete Math. 7 (1980), 215-253.

[6] M. Hall, Jr., Combinatorial Theory, Blaisdell, Waldham, Mass. (1967).

[7] H. Hanani, Balanced incomplete block designs and related designs, Discrete Math. 11 (1975), 255-369.

[8] M. Jimbo, S. Kuriki, On a composition of cyclic 2-designs, Discrete Math. 46 (1983), 249-255.

[9] P.J. Laufer, Regular perfect systems of difference sets of size 4 and extremal systems of size 3, Ann. Discrete Math. 12 (1982), 193-201.

[10] R. Peltesohn, Eine Losung der beiden Heffterschen Differenzenprobleme, Compositio Math. 6 (1939), 251-257.

[11] D.G. Rogers, Addition theorems for perfect systems of difference sets, J. Lond. Math. Soc. (2), 23 (1981), 385-395.

[12] J.M. Turgeon, Construction of additive sequences of permutations of arbitrary lengths, Ann. Discrete Math. 12 (1982), 239-242.

[13] R.M. Wilson, Cyclotomy and difference families in elementary abelian groups, $J$. Number Theory 4 (1972), 17-47.

[14] R.M. Wilson, Constructions and uses of pairwise balanced designs, Combinatorics (eds. M. Hall, Jr. and J.H. van Lint), Mathematical Centre, Amsterdam (1975), 19-42. 